\title{
Mechanochemical functionalization of mesoporous carbons for the catalytic transformation of trans-ferulic acid into vanillin
}

Noelia Lázaro ${ }^{\mathrm{a}}$, Jimena Castro-Gutiérrez ${ }^{\mathrm{b}}$, Pamela Ramírez-Vidal ${ }^{\mathrm{b}}$, Alain Celzard ${ }^{\mathrm{b}}$, Vanessa Fierro $^{\mathrm{b}}$, Tahani Saad AlGarni ${ }^{\mathrm{d}}$, Antonio Pineda ${ }^{\mathrm{a}}$ and Rafael Luque ${ }^{\mathrm{a}, \mathrm{c}^{*}}$.

a Departamento de Química Orgánica Universidad de Córdoba, Edificio Marie Curie (C-3), Campus de Rabanales, Ctra. Nnal. IV-A, Km 396, E14014, Cordoba, Spain.

b Institute Jean Lamour, UMR 7198 CNRS, University of Lorraine, ENSTIB, 27 Rue Philippe Séguin, BP 21042, 88051 Epinal Cedex 9, France.

c Peoples Friendship University of Russia (RUDN University), 6 Miklukho-Maklaya str., 117198, Moscow, Russia.

${ }^{d}$ Chemistry Department, College of Science, King Saud University, Riyadh, 11451, Saudi Arabia

*Corresponding Authors

Professor Rafael Luque, PhD (q62alsor@uco.es)

Noelia Lázaro,bt2laron@uco.es

\section{SUPPORTING INFORMATION}

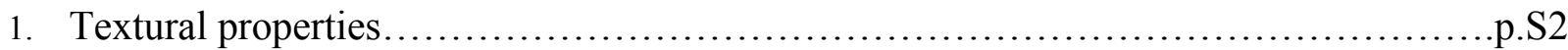

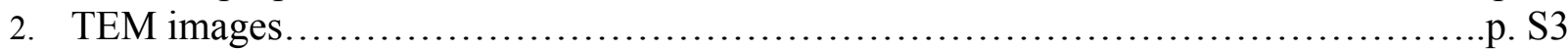

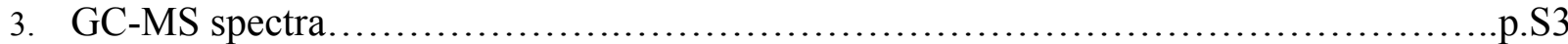

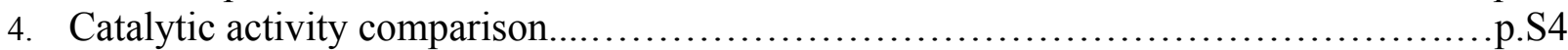




\section{Textural properties}
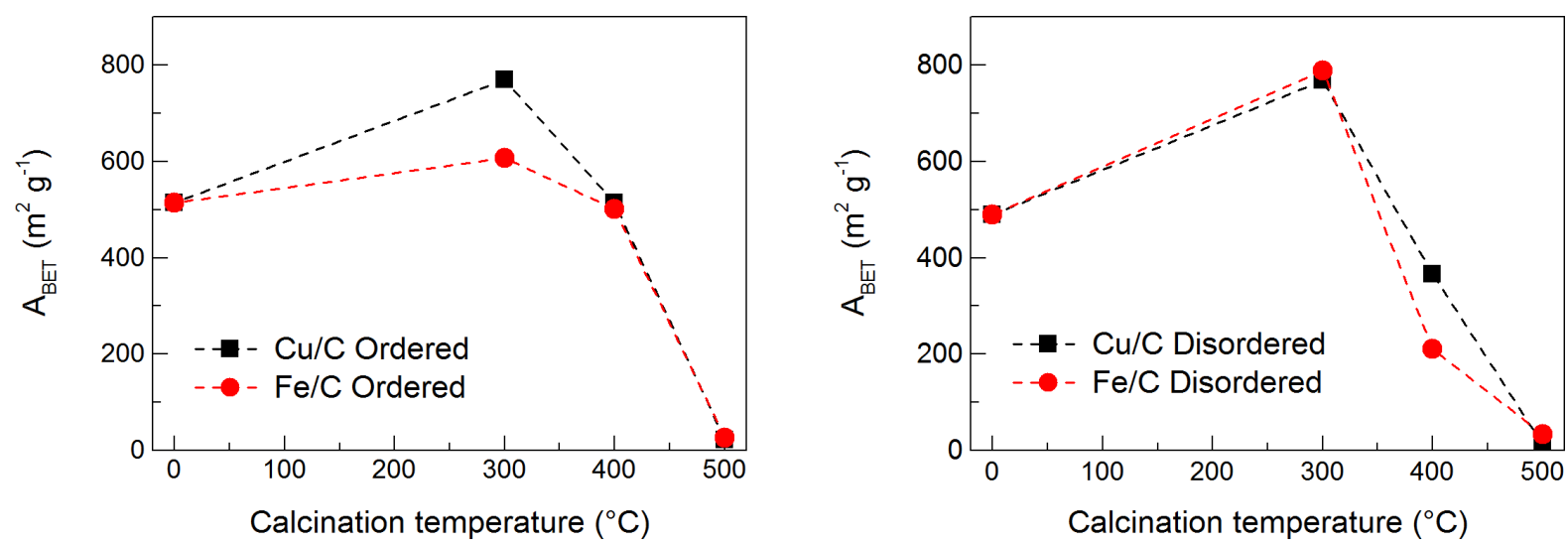

Figure $\mathrm{S} 1$. Evolution of $\mathrm{A}_{\mathrm{BET}}$ with calcination temperature.
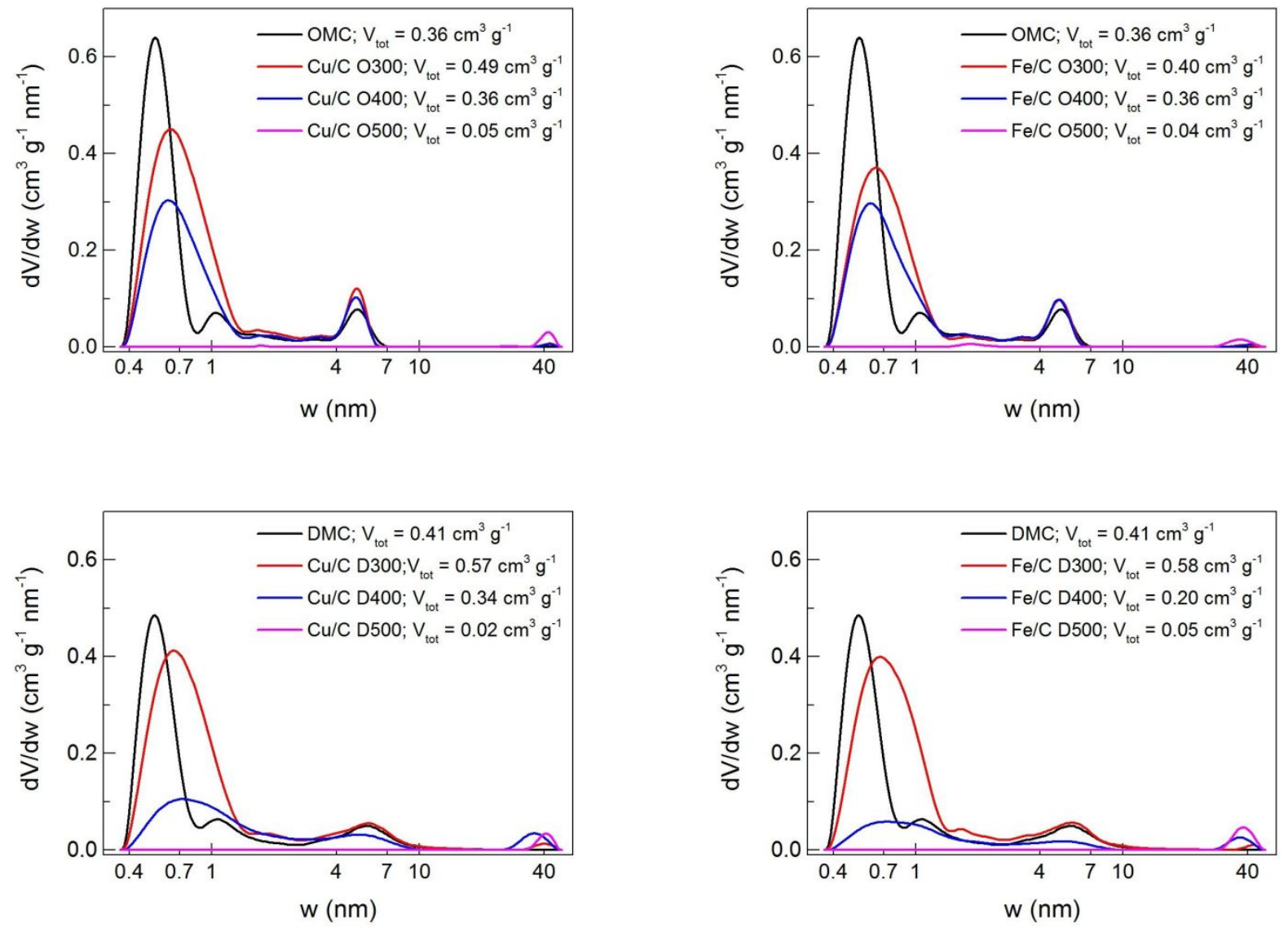

Figure S2. PSDs obtained by applying the 2D-NLDFT model to the adsorption branch of $\mathrm{N}_{2}$ isotherms. Total pore volume $\left(\mathrm{V}_{\text {tot }}\right)$ was considered as the adsorbed volume at a relative pressure of 0.97 . 


\section{TEM images}
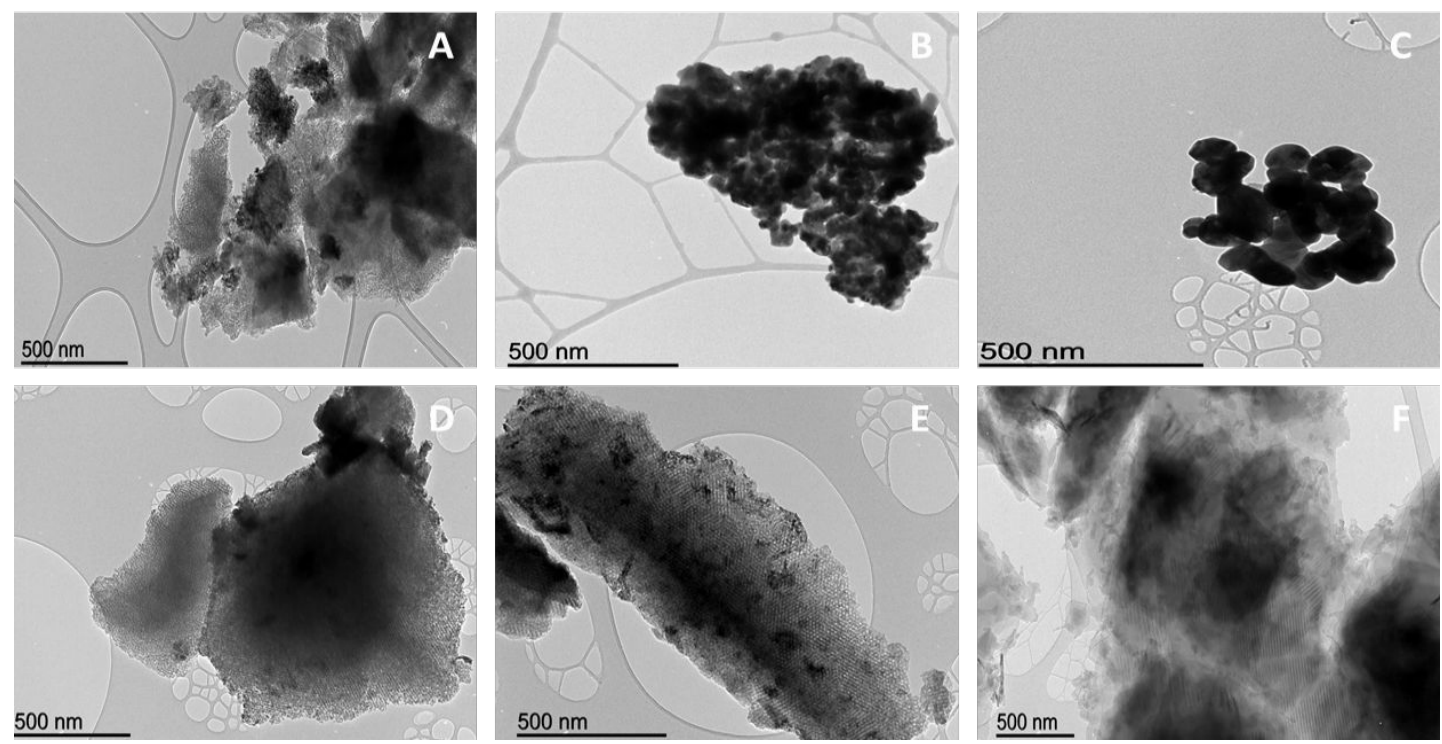

Figure S3. TEM image of synthesized materials at $500 \mathrm{~nm}$. A: $\mathrm{Cu} / \mathrm{C}$ D300; B: $\mathrm{Cu} / \mathrm{C}$ D400; C: $\mathrm{Cu} / \mathrm{C}$ D500; D: $\mathrm{Cu} / \mathrm{C} \mathrm{O} 300 ; \mathrm{E}: \mathrm{Cu} / \mathrm{C}$ O400; F: $\mathrm{Cu} / \mathrm{C} \mathrm{O} 500$.

\section{GC-MS spectra}
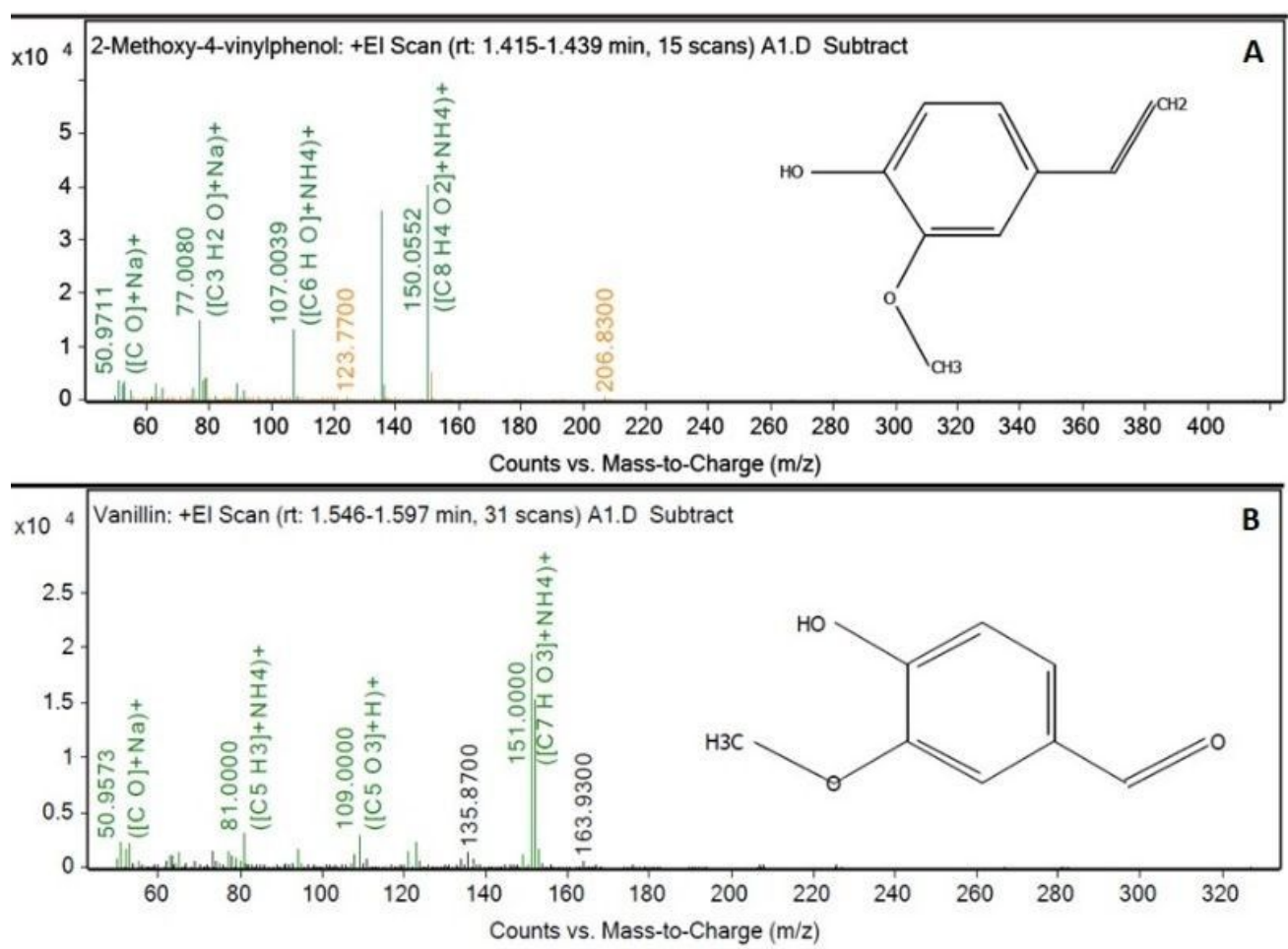
Figure S4. Products confirmation (GC-MS) in the reaction mixture. A: 2-methoxy-4-vinylphenol; B: Vanillin. 


\section{Catalytic activity}
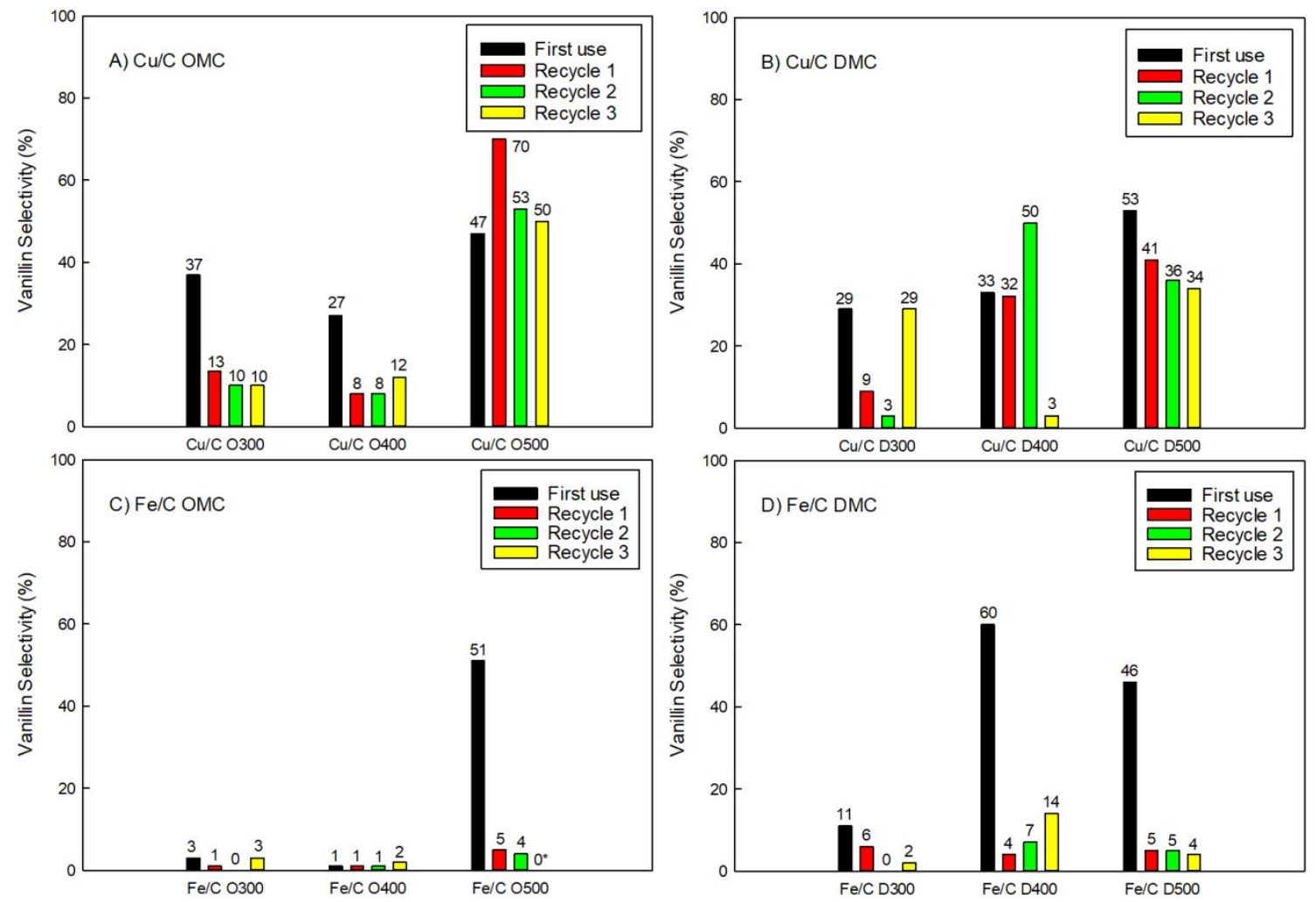

$*$ Real value $<1 \%$

Figure S5. Reusability study after 6 hour-reaction time of synthesized materials.

* 